\title{
A Family of $2 n$-Point Ternary Non-Stationary Interpolating Subdivision Scheme
}

\author{
MEHWISH BARI*, AND GHULAM MUSTAFA* \\ RECEIVED ON 19.12.2016 ACCEPTED ON 21.02.2017
}

\begin{abstract}

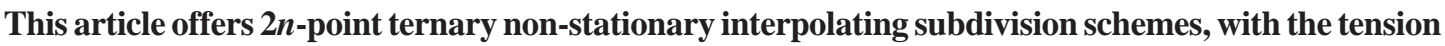
parameter, by using Lagrange identities. By choosing the suitable value of tension parameter, we can get different limit curves according to our own choice. Tightness or looseness of the limit curve depends upon the increment or decline the value of tension parameter. The proposed schemes are the counter part of some existing parametric and non-parametric stationary schemes. The main purpose of this article is to reproduce conics and the proposed schemes reproduce conics very well such that circle, ellipse, parabola and hyperbola. We also establish a deviation error formula which is useful to calculate the maximum deviation of limit curve from the original limit curve. The presentation and of the proposed schemes are verified by closed and open figures. The given table shows the less deviation of the limit curves by proposed scheme as compare to the existing scheme. Graphical representation of deviation error is also presented and it shows that as the number of control points increases, the deviation error decreases.
\end{abstract}

Key Words: Ternary Subdivision, Interpolation, Non-Stationary, Tension Control, Conics.

\section{INTRODUCTION}

ubdivision schemes are the most important, significant and emerging modeling tools in computer aided geometric design, computer applications, medical image processing and scientific visualization. It iteratively refines a given set of control points according to certain refinement rules. Several subdivision schemes for generation of curves and surfaces have been introduced in literature.

Deslauriers and Dubuc [1] define a symmetric iterative interpolation processes. Hassan et. al. [2] analyzed a novel 4-point ternary interpolatory subdivision scheme with a tension parameter. Zheng et. al. [3] devise a novel (2n-1)-point interpolatory ternary subdivision scheme that reproduces polynomial of degree $2 n-2$. Further a family of $a$-ary and (2n-1)-point ternary interpolating subdivision schemes are produced by [3-4] respectively. Siddiqi and Rehan [5] introduced 3-point ternary scheme and modified it by a tension parameter which generates family of $C^{1}$ and $C^{2}$ limiting curves for certain range of tension parameter. Siddiqi and Ahmed [6] proposed a new five point approximating subdivision scheme for

Correspondence Author (E-Mail: mehwishbari@yahoo.com)

Department of Mathematics, The Islamia University of Bahawalpur, Bahawalpur.

Mehran University Research Journal of Engineering \& Technology, Volume 36, No. 4, October, 2017 [p-ISSN: 0254-7821, e-ISSN: 2413-7219] 
the generation of smooth limiting curves. Bari et. al. [7] worked on the $3 n$-point quaternary shape preserving subdivision schemes.

The important schemes for applications should allow controlling the shape of the limit curve and being capable of reproducing families of curves widely used in Computer Graphics, such as conic sections and polynomials. Initially, stationary subdivision schemes are established but they do not have the capability to produce conics. Later on, the work on non-stationary schemes grows rapidly which can produce conics. Jena et al. [8] proposed 4-point binary non-stationary interpolating scheme. This scheme reproduces elements of the linear space spanned by $\{1, \sin (\alpha x), \cos (\alpha x)\}$. A non-stationary uniform tension controlled interpolating 4-point scheme with a single tension parameter having $C^{1}$ continuity was proposed by Beccari et. al. [9]. A 4point ternary interpolating non-stationary scheme spanned by $\{1, \sin (\alpha x), \cos (\alpha x)\}$ was proposed by Daniel and Shunmugaraj [10]. Bari and Mustafa [11] proposed a family of 4-point $n$-ary interpolating scheme. They also worked on odd-point non-stationary interpolating subdivision scheme [12]. Conti et. al. [13] introduced a new equivalence notion between non-stationary subdivision schemes, termed asymptotic similarity, which is weaker than asymptotic equivalence. Novara and Romani [14] defined the building blocks to obtain new families of non-stationary subdivision schemes. Mustafa and Ashraf [15] presented a family of 4-point odd-ary interpolating non-stationary schemes. The common criteria to evaluate the quality of a subdivision scheme are smoothness and shape preserving properties. The idea is to construct a $2 n$-point (for any integer $n \geq 2$ ) ternary interpolating scheme with the ability that the masks of the proposed schemes with suitable tension parameter converge to stationary schemes and preserve the shape of initial polygon due to interpolating behavior. Bari [16] discuss the non-stationary work.
In this paper, Section 2 presents some results which are useful to generate a class of non-stationary ternary interpolating schemes. We proposed $2 n$-point nonstationary ternary interpolating schemes in Section 3, providing the user with a tension parameter that, when increased within its range of definition, can generate continuous limit curves. It also provides the convergence of proposed interpolating schemes; such schemes repair the draw backs of its stationary analogue [1-2,12] which does not give the possibility to appreciate significant modification, such that the limit curve of stationary subdivision scheme is determined completely by its initial control mesh. So it is not suitable to alter the shape by the scheme itself. Furthermore, a stationary subdivision scheme can't produce conics, which are useful in different applications. Moreover, the limit curves formed by proposed schemes are more accurate because of interpolating behavior of schemes. In particular if the initial control points are equidistant and lie on a circle, the proposed schemes generate circle. Other conics such that ellipse, parabola and hyperbola are formed by taking the initial data points from their parametric equation and in the result after applying proposed schemes, the limit curve will be ellipse, parabola and hyperbola respectively.

\section{PRELIMINARIES}

A ternary univariate subdivision scheme is defined in terms of a mask consisting of a finite set of non-zero coefficients

$$
a^{k}=\left\{a_{i}^{k}: i \in Z\right\}
$$

The scheme, in compact form, is given by a subdivision rule:

$$
p_{i}^{k+1}=\sum_{j \in Z} a_{i-3 j}^{k} p_{i}^{k}, i \in Z
$$


If the mask $a^{k}$ is independent of $k$ the subdivision scheme $S_{a^{k}}$ corresponding to the mask $a^{k}$ is called stationary otherwise it is called non-stationary.

Definition-1: Two ternary subdivision schemes $S_{a^{k}}$ and $S_{b^{k}}$ are asymptotically equivalent if

$\sum_{k=0}^{\infty}\left\|S_{a^{k}}-S_{b^{k}}\right\|<\infty$

Where

$\left\|S_{a^{k}}\right\|_{\infty}=\max \left\{\sum_{i \in Z}\left|a_{3 i}^{k}\right|, \sum_{i \in Z}\left|a_{3 i+1}^{k}\right|, \sum_{i \in Z}\left|a_{3 i+2}^{k}\right|\right\}$

The idea behind asymptotic equivalence was presented by Dyn and Levin [17]. The proof of the following theorem follows exactly similar by [9] to the proof of the theorem given in (Theorem-8, [17]).

Theorem-1: Let $S_{a^{k}}$ and $S_{a}$ be two ternary non-stationary and stationary subdivision schemes, respectively, having finite masks of the same support. If stationary scheme $S_{a}$ is and $\sum_{k=0}^{\infty} 3^{m k}\left\|S_{a^{k}}-S_{a}\right\|_{\infty}<\infty$ then the non-stationary scheme $S_{a^{k}}$ is $C^{m}$

Construction of subdivision schemes using Lagrange interpolation was presented by Deslauriers and Dubuc [1]. We also use Lagrange polynomial to construct a class of non-stationary schemes. Here we define Lagrange fundamental polynomials of degree $2 n$ and $2 n-1$ for any integer $n \geq 2$ corresponding to nodes $\left\{x_{j}\right\}_{-(n-1)}^{n}$ and $\left\{x_{j}\right\}_{-(n-2)}^{n}$ respectively,

$L_{m}^{2 n}(x) \prod_{x_{j}=-(n-1) m \neq x_{j}}^{n} \frac{x-x_{j}}{m-x_{j}}, m=-(n-1),-(n-2), \ldots, n$

where $x_{j}=-(n-1), \ldots, n$ and

$L_{m}^{2 n}(x) \prod_{x_{j}=-(n-2) m \neq x_{j}}^{n} \frac{x-x_{j}}{m-x_{j}}, m=-(n-2),-(n-3), \ldots, n$ where $x_{j}=-(n-2), \ldots, n$.

By using algebraic operations on the fundamental Lagrange identities in Equations (1-2), we get following Equations (3-4):

$L_{m}^{2 n}\left(\frac{1}{3}\right)=\frac{\frac{(-1)^{n}(3 n-1) !}{3^{3 n-2}(1-3 m)(n-1) !}}{(-1)^{n-m}(n-m) !(n+m-1) !}$

where $m=-(n-1),-(n-2), \ldots, n$, and

$$
L_{m}^{2 n-1}\left(\frac{1}{3}\right)=\frac{\frac{(-1)^{n}(3 n-1)(3 n-4) !}{3^{3 n-4}(1-3 m)(n-2) !}}{(-1)^{n-m}(n-m) !(n+m-2) !}=\frac{\sigma_{1}}{\sigma_{2}}
$$

implies

$$
L_{m}^{2 n-1}\left(\frac{1}{3}\right)=\frac{\sigma_{1}}{\sigma_{2}} \text { for } m=-(n-2),-(n-3), \ldots, n
$$

where

$$
\sigma_{1}=\frac{(-1)^{n}(3 n-1)(3 n-4) !}{3^{3 n-4}(1-3 m)(n-2) !}
$$

and

$\sigma_{2}=(-1)^{n-m}(n-m) !(n+m-2) !$

Further, for $m=-n+1$ in Equation (3), we get

$$
\sigma_{3}=L_{m}^{2 n}\left(\frac{1}{3}\right)=L_{m}^{2 n-1}\left(\frac{1}{3}\right)=\frac{(-1)^{m}(3 n-1) !}{3^{3 n-2}(3 n-2)(n-1) !(n-m) !(n+m-1) !}
$$

Furthermore, we have

$$
\sigma_{4}=L_{m}^{2 n}\left(\frac{1}{3}\right)=L_{m}^{2 n-1}\left(\frac{1}{3}\right)=\frac{(-1)^{m}(3 n-1) !}{3^{3 n-2}(3 n-2)(n-1) !(n-m) !(n+m-1) !}
$$

where $m=-(n-2),-(n-3), \ldots, n$. For more detail, we may refer to [18]. 


\section{2N-POINTTERNARY INTERPOLATING SCHEME}

In this section, we present general explicit formulae to construct the mask of $2 n$-point ternary non-stationary interpolating subdivision scheme.

For $n \geq 2$, the mask of $2 n$-point ternary interpolating scheme is

$$
\left\{\begin{array}{c}
p_{3 i}^{k+1}=p_{i}^{k} \\
p_{3 i+1}^{k+1}=\sum_{m=-n}^{n-1} \mu_{-m}^{k, 2 n} p_{i+m+1}^{k} \\
p_{3 i+2}^{k+1}=\sum_{m=-(n-1)}^{n-1} \mu_{m}^{k, 2 n} p_{i+m}^{k}
\end{array}\right.
$$

The first weight $\mu_{-m}^{k, 2 n}$ for $m=-n$ is formed by introducing the parameter $\omega$, sine function and triadic subdivision $\frac{1}{3^{k+1}}$ in a well defined manner. The other weights $\mu_{-m}^{k, 2 n}$ for $m=-n+1, \ldots, n-1$ are formed by perturbing the Equations (5-8).

$$
\left\{\begin{array}{c}
\mu_{-n+1}^{k, 2 n}=\frac{\sin \left(\frac{\omega}{3^{k+1}}\right)}{\sin \left(\frac{1}{3^{k+1}}\right)}, \omega<1 \\
\mu_{m}^{k, 2 n}=\frac{\sin \left(\frac{\sigma_{1}}{3^{k+1}}\right)}{\sin \left(\frac{\sigma_{2}}{3^{k+1}}\right)}+\frac{\sin \left(\frac{\sigma_{4} \omega}{3^{k+1}}\right)}{\sin \left(\frac{\sigma_{3}}{3^{k+1}}\right)}, m=-n+2, \ldots, n
\end{array}\right.
$$

substituting $n=2$ in Equations (9-10), we get new 4-point ternary interpolating scheme with parameter $\omega$.

$$
\begin{aligned}
& p_{3 i}^{k+1}=p_{i}^{k} \\
& p_{3 i+1}^{k+1}=\mu_{2}^{k, 4} p_{i-1}^{k}+\mu_{1}^{k, 4} p_{i}^{k}+\mu_{0}^{k, 4} p_{i+1}^{k}+\mu_{-1}^{k, 4} p_{i+2}^{k} \\
& p_{3 i+2}^{k+1}=\mu_{-1}^{k, 4} p_{i-1}^{k}+\mu_{0}^{k, 4} p_{i}^{k}+\mu_{1}^{k, 4} p_{i+1}^{k}+\mu_{2}^{k, 4} p_{i+2}^{k}
\end{aligned}
$$

where

$$
\begin{aligned}
& \mu_{-1}^{k, 4}=\frac{\sin \left(\frac{\omega}{3^{k+1}}\right)}{\sin \left(\frac{1}{3^{k+1}}\right)} \\
& \mu_{0}^{k, 4}=\frac{\sin \left(\frac{10}{9.3^{k+1}}\right)}{\sin \left(\frac{2}{3^{k+1}}\right)}-\frac{\sin \left(\frac{5 \omega}{27.3^{k+1}}\right)}{\sin \left(\frac{5}{81.3^{k+1}}\right)} \\
& \mu_{1}^{k, 4}=\frac{\sin \left(\frac{5}{9.3^{k+1}}\right)}{\sin \left(\frac{1}{3^{k+1}}\right)}+\frac{\sin \left(\frac{5 \omega}{27.3^{k+1}}\right)}{\sin \left(\frac{5}{81.3^{k+1}}\right)}
\end{aligned}
$$

and

$\mu_{2}^{k, 4}=-\frac{\sin \left(\frac{2}{9.3^{k+1}}\right)}{\sin \left(\frac{2}{3^{k+1}}\right)}-\frac{\sin \left(\frac{5 \omega}{81.3^{k+1}}\right)}{\sin \left(\frac{5}{81.3^{k+1}}\right)}$

Next, we will prove that the scheme converges and is $C^{2}$ Now we introduce the normalized scheme (corresponding to Equation (11)).

$\mu_{1}^{k, 4}+\mu_{0}^{k, 4}+\mu_{3}^{k, 4}+\mu_{2}^{k, 4}=\frac{\sin \left(\frac{\omega}{3^{k+1}}\right)}{\sin \left(\frac{1}{3^{k+1}}\right)}+\frac{\sin \left(\frac{10}{9.3^{k+1}}\right)}{\sin \left(\frac{2}{3^{k+1}}\right)}+\frac{\sin \left(\frac{5}{9.3^{k+1}}\right)}{\sin \left(\frac{1}{3^{k+1}}\right)}-\frac{\sin \left(\frac{2}{9.3^{k+1}}\right)}{\sin \left(\frac{2}{3^{k+1}}\right)}-\frac{\sin \left(\frac{5 \omega}{81.3^{k+1}}\right)}{\sin \left(\frac{5}{81.3^{k+1}}\right)}=\chi^{k, 4}$

The normalized scheme is defined as follows:

$$
\begin{aligned}
& p_{3 i}^{k+1}=p_{i}^{k} \\
& p_{3 i+1}^{k+1}=v_{2}^{k, 4} p_{i-1}^{k}+v_{1}^{k, 4} p_{i}^{k}+v_{0}^{k, 4} p_{i+1}^{k}+v_{-1}^{k, 4} p_{i+2}^{k} \\
& p_{3 i+2}^{k+1}=v_{-1}^{k, 4} p_{i-1}^{k}+v_{0}^{k, 4} p_{i}^{k}+v_{1}^{k, 4} p_{i+1}^{k}+v_{2}^{k, 4} p_{i+2}^{k}
\end{aligned}
$$

where

$$
v_{i}^{k, 4}=\frac{\mu_{i}^{k, 4}}{\chi^{k, 4}}, \quad \mathrm{i}=-1,0,1,2
$$

Note that the sum of coefficients of normalized scheme is equal to one. 
Substituting $n=3$ in Equations (9-10), we get new 6-point ternary interpolating scheme with free parameter $\omega$

$p_{3 i}^{k+1}=p_{i}^{k}$

$p_{3 i+1}^{k+1}=\mu_{3}^{k, 6} p_{i-2}^{k}+\mu_{2}^{k, 6} p_{i-1}^{k}+\mu_{1}^{k, 6} p_{i}^{k}+\mu_{0}^{k, 6} p_{i+1}^{k}+\mu_{-1}^{k, 6} p_{i+2}^{k}+\mu_{-2}^{k, 6} p_{i+3}^{k}$

$p_{3 i+2}^{k+1}=\mu_{-2}^{k, 6} p_{i-2}^{k}+\mu_{-1}^{k, 6} p_{i-1}^{k}+\mu_{0}^{k, 6} p_{i}^{k}+\mu_{1}^{k, 6} p_{i+1}^{k}+\mu_{2}^{k, 6} p_{i+2}^{k}+\mu_{3}^{k, 6} p_{i+3}^{k}$

where

$\mu_{-2}^{k, 6}=\frac{\sin \left(\frac{\omega}{3^{k+1}}\right)}{\sin \left(\frac{1}{3^{k+1}}\right)}$

$\mu_{-1}^{k, 6}=-\frac{\sin \left(\frac{80}{81.3^{k+1}}\right)}{\sin \left(\frac{24}{3^{k+1}}\right)}-\frac{\sin \left(\frac{40 \omega}{729.3^{k+1}}\right)}{\sin \left(\frac{8}{729.3^{k+1}}\right)}$

$\mu_{0}^{k, 6}=\frac{\sin \left(\frac{320}{81.3^{k+1}}\right)}{\sin \left(\frac{6}{3^{k+1}}\right)}+\frac{\sin \left(\frac{80 \omega}{729.3^{k+1}}\right)}{\sin \left(\frac{8}{729.3^{k+1}}\right)}$

$\mu_{1}^{k, 6}=\frac{\sin \left(\frac{160}{81.3^{k+1}}\right)}{\sin \left(\frac{4}{3^{k+1}}\right)}-\frac{\sin \left(\frac{80 \omega}{729.3^{k+1}}\right)}{\sin \left(\frac{8}{729.3^{k+1}}\right)}$

$\mu_{2}^{k, 6}=\frac{\sin \left(\frac{64}{81.3^{k+1}}\right)}{\sin \left(\frac{6}{3^{k+1}}\right)}+\frac{\sin \left(\frac{40 \omega}{729.3^{k+1}}\right)}{\sin \left(\frac{8}{729.3^{k+1}}\right)}$

and

$\mu_{3}^{k, 6}=\frac{\sin \left(\frac{40}{81.3^{k+1}}\right)}{\sin \left(\frac{24}{3^{k+1}}\right)}-\frac{\sin \left(\frac{8 \omega}{729.3^{k+1}}\right)}{\sin \left(\frac{8}{729.3^{k+1}}\right)}$.

The normalized scheme (corresponding to Equation (15)).

$\mu_{-2}^{k, 6}+\mu_{-1}^{k, 6}+\mu_{0}^{k, 6}+\mu_{1}^{k, 6}+\mu_{2}^{k, 6}+\mu_{3}^{k, 6}=\frac{\sin \left(\frac{\omega}{3^{k+1}}\right)}{\sin \left(\frac{1}{3^{k+1}}\right)}-\frac{\sin \left(\frac{80}{81.3^{k+1}}\right)}{\sin \left(\frac{24}{3^{k+1}}\right)}+\frac{\sin \left(\frac{320}{81.3^{k+1}}\right)}{\sin \left(\frac{6}{3^{k+1}}\right)}+\frac{\sin \left(\frac{160}{81.3^{k+1}}\right)}{\sin \left(\frac{4}{3^{k+1}}\right)}-$

$$
\frac{\sin \left(\frac{64}{81.3^{k+1}}\right)}{\sin \left(\frac{6}{3^{k+1}}\right)}+\frac{\sin \left(\frac{40}{81.3^{k+1}}\right)}{\sin \left(\frac{24}{3^{k+1}}\right)}-\frac{\sin \left(\frac{8 \omega}{729.3^{k+1}}\right)}{\sin \left(\frac{8}{729.3^{k+1}}\right)}=\chi^{k, 6} .
$$

The normalized scheme is defined as follows:

$$
\begin{aligned}
& p_{3 i}^{k+1}=p_{i}^{k}, \\
& p_{3 i+1}^{k+1}=v_{3}^{k, 6} p_{i-2}^{k}+v_{2}^{k, 6} p_{i-1}^{k}+v_{1}^{k, 6} p_{i}^{k}+v_{0}^{k, 6} p_{i+1}^{k}+v_{-1}^{k, 6} p_{i+2}^{k}+v_{-2}^{k, 6} p_{i+3}^{k}, \\
& p_{3 i+2}^{k+1}=v_{-2}^{k, 6} p_{i-2}^{k}+v_{-1}^{k, 6} p_{i-1}^{k}+v_{0}^{k, 6} p_{i}^{k}+v_{1}^{k, 6} p_{i+1}^{k}+v_{2}^{k, 6} p_{i+2}^{k}+v_{3}^{k, 6} p_{i+3}^{k},
\end{aligned}
$$

where

$v_{i}^{k, 6}=\frac{\mu_{i}^{k, 6}}{\chi^{k, 6}}, \quad i=-2,-1,0,1,2,3$.

Note that the sum of coefficients of normalized scheme is equal to one.

Remark-1: The general form for the weights of normalized schemes for $n \geq 2$ can be written as:

$v_{i}^{k, 2 n}=\frac{\mu_{i}^{k, 2 n}}{\chi^{k, 2 n}}, \quad i=-n+1, \ldots, n$

\subsection{Convergence of 4- and 6-Point Ternary Schemes}

By using the inequalities $\frac{\sin a}{\sin b} \geq \frac{a}{b}$ for $0<a \leq b<\frac{\pi}{2}$, $a \csc a<b s c b$ for $0<a<b<\frac{\pi}{2}$ and $\cos a<\frac{\sin a}{a}\left(\right.$ or $\left.\csc a<\frac{1}{a \cos a}\right)$ for $0<a<\frac{\Pi}{2}$, we will show that proposed non-stationary schemes are asymptotically equivalent to existing stationary schemes.

\section{Lemma-1:}

For 4-point non-stationary scheme Equation (13) following inequalities hold:

$$
\omega \leq v_{-1}^{k, 4} \leq \frac{\omega \cos \left(\frac{5}{81.3^{k+1}}\right)}{\cos \left(\frac{1}{3^{k+1}}\right)}
$$

(ii) 
(iii)

$$
\begin{aligned}
& \frac{5}{9}+3 \omega \cos \left(\frac{5 \omega}{27.3^{k+1}}\right) \leq v_{1}^{k, 4} \leq \frac{5}{9}+3 \omega \\
& -\frac{1}{9 \cos \left(\frac{2}{3^{k+1}}\right)}-\frac{\omega}{\cos \left(\frac{5}{81.3^{k+1}}\right)} \leq v_{2}^{k, 4} \leq\left(-\frac{1}{9}-\omega\right) \cos \left(\frac{5}{81.3^{k+1}}\right)
\end{aligned}
$$

Proof. We present the proof of (i) and the proof of (ii), (iii) and (iv) are similar.

$$
v_{-1}^{k, 4}=\frac{\mu_{-1}^{k, 4}}{\chi^{k, 4}}=\frac{\frac{\sin \left(\frac{\omega}{3^{k+1}}\right)}{\sin \left(\frac{1}{3^{k+1}}\right)}}{\frac{\sin \left(\frac{\omega}{3^{k+1}}\right)}{\sin \left(\frac{1}{3^{k+1}}\right)}+\frac{\sin \left(\frac{10}{9.3^{k+1}}\right)}{\sin \left(\frac{2}{3^{k+1}}\right)}+\frac{\sin \left(\frac{5}{9.3^{k+1}}\right)}{\sin \left(\frac{1}{3^{k+1}}\right)}-\frac{\sin \left(\frac{2}{9.3^{k+1}}\right)}{\sin \left(\frac{2}{3^{k+1}}\right)}-\frac{\sin \left(\frac{5 \omega}{81.3^{k+1}}\right)}{\sin \left(\frac{5}{81.3^{k+1}}\right)}}
$$

Again consider

$$
\begin{aligned}
& \geq \frac{\frac{\frac{\omega}{3^{k+1}}}{\frac{1}{3^{k+1}}}}{\frac{\frac{\omega}{3^{k+1}}}{\frac{1}{3^{k+1}}}+\frac{\frac{10}{9.3^{k+1}}}{\frac{2}{3^{k+1}}}+\frac{\frac{5}{9.3^{k+1}}}{\frac{1}{3^{k+1}}}-\frac{\frac{2}{9.3^{k+1}}}{\frac{2}{3^{k+1}}}-\frac{\frac{5 \omega}{81.3^{k+1}}}{\frac{5}{81.3^{k+1}}}} \geq \omega . \\
& v_{-1}^{k, 4}=\frac{\frac{\sin \left(\frac{\omega}{3^{k+1}}\right)}{\sin \left(\frac{1}{3^{k+1}}\right)}}{\frac{\sin \left(\frac{\omega}{3^{k+1}}\right)}{\sin \left(\frac{1}{3^{k+1}}\right)}+\frac{\sin \left(\frac{10}{9.3^{k+1}}\right)}{\sin \left(\frac{2}{3^{k+1}}\right)}+\frac{\sin \left(\frac{5}{9.3^{k+1}}\right)}{\sin \left(\frac{1}{3^{k+1}}\right)}-\frac{\sin \left(\frac{2}{9.3^{k+1}}\right)}{\sin \left(\frac{2}{3^{k+1}}\right)}-\frac{\sin \left(\frac{5 \omega}{81.3^{k+1}}\right)}{\sin \left(\frac{5}{81.3^{k+1}}\right)}} \\
& \leq \frac{\frac{\omega}{\cos \left(\frac{1}{3^{k+1}}\right)}}{\frac{\omega}{\cos \left(\frac{1}{3^{k+1}}\right)}+\frac{4}{9 \cos \left(\frac{2}{3^{k+1}}\right)}+\frac{5}{9 \cos \left(\frac{1}{3^{k+1}}\right)}-\frac{\omega}{\cos \left(\frac{5}{81.3^{k+1}}\right)}} \\
& \leq \frac{\frac{\omega}{\cos \left(\frac{1}{3^{k+1}}\right)}}{\frac{\omega}{\cos \left(\frac{5}{81.3^{k+1}}\right)}+\frac{4}{9 \cos \left(\frac{5}{81.3^{k+1}}\right)}+\frac{5}{9 \cos \left(\frac{5}{81.3^{k+1}}\right)}-\frac{\omega}{\cos \left(\frac{5}{81.3^{k+1}}\right)}} \leq \frac{\omega \cos \left(\frac{5}{81.3^{k+1}}\right)}{\cos \left(\frac{1}{3^{k+1}}\right)}
\end{aligned}
$$

This proves(i).

From Lemma-1, we get following lemma.

\section{Lemma-2:}

For scheme Equation (13) with $\omega=-\frac{1}{18}-\frac{1}{6} u$, for $u \in\left(\frac{1}{15}, \frac{1}{9}\right)$, we have (i)

$$
\begin{aligned}
& -\frac{1}{18}-\frac{1}{6} u \leq v_{-1}^{k, 4} \leq \frac{\left(-\frac{1}{15}-\frac{1}{6} u\right) \cos \left(\frac{5}{81.3^{k+1}}\right)}{\cos \left(\frac{1}{3^{k+1}}\right)} \\
& \frac{5}{9}-3 \frac{\left(-\frac{1}{18}-\frac{1}{6} u\right)}{\cos \left(\frac{5}{81.3^{k+1}}\right)} \leq v_{0}^{k, 4} \leq \frac{13}{18}+\frac{1}{6} u
\end{aligned}
$$

(iii)

$$
\begin{aligned}
& \frac{5}{9}+3\left(-\frac{1}{18}-\frac{1}{6} u\right) \cos \frac{\left(-\frac{5}{18}-\frac{5}{6} u\right)}{81.3^{k+1}} \leq v_{1}^{k, 4} \leq \frac{7}{18}-\frac{1}{6} u \\
& -\frac{1}{9 \cos \left(\frac{2}{3^{k+1}}\right)}-\frac{\left(-\frac{1}{18}-\frac{1}{6} u\right)}{\cos \left(\frac{5}{81.3^{k+1}}\right)} \leq v_{2}^{k, 4} \leq\left(-\frac{1}{18}+\frac{1}{6} u\right) \cos \left(\frac{5}{81.3^{k+1}}\right)
\end{aligned}
$$

\section{Lemma-3:}

For scheme Equation (13) following inequalities also hold:

$$
\begin{aligned}
& \left|v_{-1}^{k, 4}-\omega\right| \leq C_{0}\left(\frac{1}{3^{2 k}}\right) \\
& \left|v_{0}^{k, 4}-\left(\frac{5}{9}-\frac{3 \omega}{\cos \left(\frac{5}{81.3^{k+1}}\right)}\right)\right| \leq C_{1}\left(\frac{1}{3^{2 k}}\right) \\
& \left|v_{1}^{k, 4}-\left(\frac{5}{9}+3 \omega \cos \left(\frac{5 \omega}{27.3^{k+1}}\right)\right)\right| \leq C_{2}\left(\frac{1}{3^{2 k}}\right)
\end{aligned}
$$

(iv)

$$
\left|v_{2}^{k, 4}-\left(-\frac{1}{9 \cos \left(\frac{2}{3^{k+1}}\right)}-\frac{\omega}{\cos \left(\frac{5}{81.3^{k+1}}\right)}\right)\right| \leq C_{3}\left(\frac{1}{3^{2 k}}\right)
$$

where constants $C_{0}, C_{1}, C_{2}$, and $C_{3}$ are independent of $k$.

Proof. The inequality (i)can be proved by using (i) of Lemma-1 and using the trigonometric identities, $\cos a-\cos b=-2 \sin \frac{a+b}{2} \sin \frac{a-b}{2}$ and $\sin a \leq a$ : 
$\left|\nu_{-1}^{k, 4}-\omega\right|=\omega\left[\frac{\cos \left(\frac{5}{81.3^{k+1}}\right)-\cos \left(\frac{1}{3^{k+1}}\right)}{\cos \left(\frac{1}{3^{k+1}}\right)}\right] \leq \omega\left[\frac{2 \sin \left(\frac{38}{81} \frac{1}{3^{k+1}}\right) \sin \left(\frac{43}{81} \frac{1}{3^{k+1}}\right)}{\cos \left(\frac{1}{3^{k+1}}\right)}\right] \leq\left(\frac{3268}{6561}\right)\left(\frac{\omega}{3^{2 k+2} \cos \left(\frac{1}{3^{k+1}}\right)}\right)$

This implies

$\left|v_{-1}^{k, 4}-\omega\right| \leq\left(\frac{1}{3^{2 k}}\right)\left(\frac{3268 \omega}{59049 \cos (1)}\right) \leq C_{0}\left(\frac{1}{3^{2 k}}\right)$

The proofs of (iii), and (iv) are similar.

From Lemma-3, we get following lemma.

\section{Lemma-4.}

For scheme Equation (13) with, $\omega=-\frac{1}{18}-\frac{1}{6} u$, for $u \in\left(\frac{1}{15}, \frac{1}{9}\right)$, for following inequalities hold:

$$
\left|v_{-1}^{k, 4}-\left(-\frac{1}{18}-\frac{1}{6} u\right)\right| \leq \mathrm{C}_{0}^{\prime}\left(\frac{1}{3^{2 k}}\right)
$$

(ii)

$$
\mid v_{0}^{k, 4}-\left(\frac{5}{9}-3 \frac{\left(-\frac{1}{18}-\frac{1}{6} u\right)}{\cos \left(\frac{5}{81.3^{k+1}}\right)} \mid \leq \mathrm{C}_{1}^{\prime}\left(\frac{1}{3^{2 k}}\right)\right.
$$

$$
\mid v_{1}^{k, 4}-\left(\frac{5}{9}+3\left(-\frac{1}{18}-\frac{1}{6} u\right) \cos \left(\frac{-\frac{5}{18}-\frac{5}{6} u}{81.3^{k+1}}\right) \mid \leq \mathrm{C}_{2}^{\prime}\left(\frac{1}{3^{2 k}}\right)\right.
$$

$$
\mid v_{2}^{k, 4}-\left(-\frac{1}{9 \cos \left(\frac{2}{3^{k+1}}\right)}-\frac{\left(-\frac{1}{18}-\frac{1}{6} u\right)}{\cos \left(\frac{5}{18.3^{k+1}}\right)} \mid \leq \mathrm{C}_{3}^{\prime}\left(\frac{1}{3^{2 k}}\right)\right.
$$

where constants $C_{0}^{\prime}, C_{1}^{\prime}, C_{2}^{\prime}$ and $C_{3}^{\prime}$ are independent of $k$.

Remark-2. From (i-iv) of Lemma-4, we observe that

$$
\mathrm{v}_{-1}^{k, 4} \rightarrow-\frac{1}{18}-\frac{1}{6} u, \mathrm{v}_{=}^{k, 4} \rightarrow-\frac{13}{18}-\frac{1}{2} u, \mathrm{v}_{1}^{k, 4} \rightarrow-\frac{7}{18}-\frac{1}{2} u, \text { and } \mathrm{v}_{2}^{k, 4} \rightarrow-\frac{1}{18}+\frac{1}{6} u, \text { as } k \rightarrow \infty
$$

This means that the mask of the scheme Equation (13) with $\omega=-\frac{1}{18}-\frac{1}{6} u$, for $u \in\left(\frac{1}{15}, \frac{1}{9}\right)$ converge to the mask of the scheme [2].

Similarly, for $\omega=-\frac{5}{18}$, in Equations (9-10) and by proving/ using similar inequalities like in Lemma-1 and Lemma-3, we get non-stationary counter part of stationary schemes of [1] respectively.

Theorem-2: The proposed 4-point non-stationary scheme Equation (13) with $\omega=-\frac{1}{18}-\frac{1}{6} u, u \in\left(\frac{1}{15}, \frac{1}{9}\right)$ is $\mathrm{C}^{2}$.

Proof. We claim that

$\sum_{k=0}^{\infty} 3^{2 k}\left\|S_{a^{a}}-S_{a}\right\|_{\infty}<\infty$

where

$$
\left\|S_{a^{k}}-S_{a}\right\|_{\infty}=\max \left\{\sum_{j \in Z}\left|a_{i-3 j}^{k}-a_{i-3 j}\right|: i \in 0,1,2,3\right\}
$$

From scheme $S_{a^{k}}$ defined by Equation (13) with $\omega=-\frac{1}{18}-\frac{1}{6} u$ and scheme $S_{a}$ of [2] (also see the Remark-2)

$$
\sum_{k=0}^{\infty} 3^{2 k}\left\|S_{a^{k}}-S_{a}\right\|_{\infty}=\sum_{k=0}^{\infty} 3^{2 k}\left\{\begin{array}{l}
\left|v_{-1}^{k, 4}-\left(-\frac{1}{18}-\frac{1}{6} u\right)\right|+\left|v_{0}^{k, 1}-\left(\frac{13}{18}+\frac{1}{2} u\right)\right|+ \\
\left|v_{1}^{k, 4}-\left(\frac{7}{18}-\frac{1}{2} u\right)\right|+\left|v_{2}^{k, 4}+\left(-\frac{1}{18}-\frac{1}{6} u\right)\right|
\end{array}\right\}
$$

From (i) of Lemma-4, it follows that:

$$
\sum_{k=0}^{\infty} 3^{2 k}\left|v_{-1}^{k, 4}-\left(-\frac{1}{18}-\frac{1}{6} u\right)\right| \leq \sum_{k=0}^{\infty} 3^{2 k} C_{0}\left(\frac{1}{3^{2 k}}\right)<\infty
$$

Similarly from (ii), (iii) and (iv) of Lemma-4, we see that other terms are also less than $\infty$. Hence $\sum_{k=0}^{\infty} 3^{2 k}\left\|S_{a^{k}}-S_{a}\right\|_{\infty}<\infty$. Since stationary scheme of [2] is $C^{2}$ therefore by Theorem2, proposed scheme Equation (13) with $\omega=-\frac{1}{81}-\frac{1}{6} u$ is $\mathrm{C}^{2}$. 
Now we will discuss the continuity of 6-point scheme

Equation (17). For this first we will prove the following lemmas. Proof of these lemmas is similar to the proof of Lemmas-1-4.

Lemma-5:

For 6-point non-stationary scheme Equation (17), following inequalities hold:

$$
\omega \leq v_{-2}^{k, 6} \leq \frac{\omega}{\cos \left(\frac{1}{3^{k+1}}\right)}
$$

$$
-\frac{10}{243}-5 \omega \leq v_{-1}^{k, 6} \leq \frac{-\frac{10}{243}-5 \omega}{\cos \left(\frac{8}{729.3^{k+1}}\right)}
$$

$$
\frac{160}{243}+10 \omega \leq v_{0}^{k, 6} \leq \frac{\frac{160}{243}+10 \omega}{\cos \left(\frac{6}{3^{k+1}}\right)}
$$

$$
\frac{40}{81}-10 \omega \leq v_{1}^{k, 6} \leq \frac{\frac{40}{81}}{\cos \left(\frac{4}{3^{k+1}}\right)}-\frac{10 \omega}{\cos \left(\frac{8}{729.3^{k+1}}\right)}
$$

$$
\begin{aligned}
& -\frac{32}{243}+5 \omega \leq v_{2}^{k, 6} \leq \frac{-\frac{32}{243}+5 \omega}{\cos \left(\frac{8}{729.3^{k+1}}\right)} \\
& \frac{5}{243}-\omega \leq v_{3}^{k, 6} \leq \frac{5}{243 \cos \left(\frac{24}{3^{k+1}}\right)}-\frac{\omega}{\cos \left(\frac{8}{729.3^{k+1}}\right)}
\end{aligned}
$$

From Lemma-5, we get following lemma:

\section{Lemma-6:}

For scheme Equation (17) with $\omega=\frac{5}{243}-\vartheta$, for $\vartheta \in\left(\frac{7}{972}, \frac{11}{1215}\right)$, we have

$$
\frac{5}{243}-\vartheta \leq v_{-2}^{k, 6} \leq \frac{\frac{5}{243}-\vartheta}{\cos \left(\frac{1}{3^{k+1}}\right)}
$$

$$
-\frac{35}{243}+5 \vartheta \leq v_{-1}^{k, 6} \leq \frac{-\frac{35}{243}+5 \vartheta}{\cos \left(\frac{8}{729.3^{k+1}}\right)}
$$

(iii)

$$
\frac{70}{81}-10 \vartheta \leq v_{0}^{k, 6} \leq \frac{\frac{70}{81}-10 \vartheta}{\cos \left(\frac{6}{3^{k+1}}\right)}
$$

(iv)

$$
\frac{70}{243}+10 \vartheta \leq v_{1}^{k, 6} \leq \frac{\frac{40}{81}}{\cos \left(\frac{4}{3^{k+1}}\right)}-\frac{10\left(\frac{5}{243}-\vartheta\right)}{\cos \left(\frac{8}{729.3^{k+1}}\right)}
$$

(v)

$$
-\frac{7}{243}-5 \vartheta \leq v_{2}^{k, 6} \leq \frac{-\frac{7}{243}-5 \vartheta}{\cos \left(\frac{8}{729.3^{k+1}}\right)}
$$

$$
\vartheta \leq v_{3}^{k, 6} \leq \frac{5}{243 \cos \left(\frac{24}{3^{k+1}}\right)}-\frac{\frac{5}{243}-\vartheta}{\cos \left(\frac{8}{729.3^{k+1}}\right)}
$$

\section{Lemma-7:}

For scheme Equation (17) and from Lemma-5, following inequalities also hold:

(i) $\quad\left|v_{-2}^{k, 6}-\omega\right| \leq g_{0}\left(\frac{1}{3^{2 k}}\right)$

(ii)

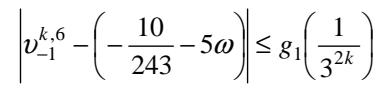

(iii)

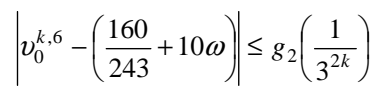

(iv)

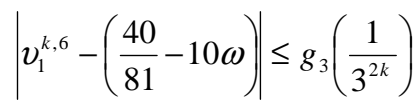

(v)

$\left|v_{2}^{k, 6}-\left(-\frac{32}{243}+5 \omega\right)\right| \leq g_{4}\left(\frac{1}{3^{2 k}}\right)$

(vi)

$$
\left|v_{3}^{k, 6}-\left(\frac{5}{243}+\omega\right)\right| \leq g_{5}\left(\frac{1}{3^{2 k}}\right)
$$

where constants $g_{0}, g_{1}, g_{2}, g_{3}, g_{4}$, and $g_{5}$ are independent of $k$.

From Lemma-7, we get following lemma.

\section{Lemma-8:}

For scheme Equation (17) with $\omega=\frac{5}{243}-\vartheta$, for $\vartheta \in\left(\frac{7}{972}, \frac{11}{1215}\right)$ and from Lemma-6, following inequalities hold: 
(i) $\quad\left|v_{-2}^{k, 6}-\left(\frac{5}{243}-\vartheta\right)\right| \leq g_{0}^{\prime}\left(\frac{1}{3^{2 k}}\right)$

(ii) $\quad\left|v_{-1}^{k, 6}-\left(\frac{35}{243}+5 \vartheta\right)\right| \leq g_{1}^{\prime}\left(\frac{1}{3^{2 k}}\right)$

(iii) $\quad\left|v_{0}^{k, 6}-\left(\frac{70}{81}-10 \vartheta\right)\right| \leq g_{2}^{\prime}\left(\frac{1}{3^{2 k}}\right)$

(iv) $\quad\left|v_{1}^{k, 6}-\left(\frac{70}{243}-10 \vartheta\right)\right| \leq g_{3}^{\prime}\left(\frac{1}{3^{2 k}}\right)$

(v) $\quad\left|v_{2}^{k, 6}-\left(-\frac{7}{243}-5 \vartheta\right)\right| \leq g_{4}^{\prime}\left(\frac{1}{3^{2 k}}\right)$

(vi)

$$
\left|v_{3}^{k, 6}-\vartheta\right| \leq g_{5}^{\prime}\left(\frac{1}{3^{2 k}}\right)
$$

where constants $g_{0}^{\prime}, g_{1}^{\prime}, g_{2}^{\prime} \cdot g_{3}^{\prime}, g_{4}^{\prime}$ and $g_{5}^{\prime}$ are independent of $k$.

Remark-3: It is to be noted that for negative values of $\omega$ the trigonometric inequalities do not affect the proof of our main Lemmas-3 and 7.

Remark-4: From (i-vi) of Lemma-8, we observe that the mask of scheme Equation (17) with $\omega=\frac{5}{243}-\vartheta, \vartheta \in\left(\frac{7}{972}, \frac{11}{1215}\right)$ converges to the mask of the scheme $S_{a}$ of [11].

$v_{-2}^{k, 6} \rightarrow \frac{5}{243}-\vartheta, v_{-1}^{k, 6} \rightarrow-\frac{35}{243}+5 \vartheta, v_{0}^{k, 6} \rightarrow \frac{70}{81}-10 \vartheta, v_{1}^{k, 6} \rightarrow \frac{70}{273}+10 \vartheta, v_{2}^{k, 6} \rightarrow-\frac{7}{243}-5 \vartheta$

and

$v_{3}^{k, 6} \rightarrow \vartheta$, as $k \rightarrow \infty$

Similarly, for $n=3, \omega=\frac{8}{729}$ in Equations (9-10) and using similar inequalities as in Lemma-5 and Lemma-7, the proposed Equation (17) scheme becomes non-stationary counterpart of the 6-point stationary schemes of [1] respectively.

Theorem 3.10. The proposed 6-point non-stationary scheme Equation (17) with $\omega=\frac{5}{243}-\vartheta$, for $\vartheta \in\left(\frac{7}{972}, \frac{11}{1215}\right)$ is $C^{2}$.
Proof. From schemedefined by Equation (17) with $\omega=\frac{5}{243}-\vartheta, \vartheta \in\left(\frac{7}{972}, \frac{11}{1215}\right)$ and scheme $S_{a}$ of [1] (also see the Remark-4)

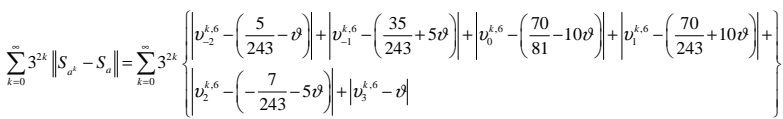

By using inequalities (i-v) of Equation (17), we see that $\sum_{k=0}^{\infty} 3^{2 k}\left\|S_{a^{k}}-S_{a}\right\|_{\infty}<\infty$.

Since stationary scheme of [17] is $C^{2}$ therefore by Theorem-2, proposed scheme Equation (17) is $C^{2}$.

\subsection{Comparison}

If the initial control points are chosen as the values at equidistant points of a function $\mathrm{f}(\mathrm{x}) \in \operatorname{span}\{\cos (\beta \mathrm{x})$, $\sin (\beta \mathrm{x})\}, 0<\beta<\pi$, then the limit function of the scheme is the original function. In particular, if the initial control points are equidistant points and lie on a circle, the scheme generates a circle. For example we can take the set of equidistant points $p_{i}^{0}=\left(a \cos \left(\frac{2 m \pi}{N}\right), b \sin \left(\frac{2 m \pi}{N}\right)\right), i \in Z$ and $m=0.1 .2 \ldots, N, N \geq 4$, it gives initial control polygons to check the behavior of proposed 4-, 6-point non-stationary schemes.

We can compare the exactness of limiting circles generated by different non-stationary subdivision schemes by using following distance function.

$d_{k}=\max _{i}\left|p_{i}^{k}-\hat{O}\right|-\min _{i}\left|p_{i}^{k}-\hat{O}\right|, \quad i \in Z$

where $p_{i}^{k}$ are control points generated by subdivision scheme at $k$-th level of iteration for $k>0$ and $\hat{O}$ is the origin of circle. The deviation error $\mathrm{d}_{0}$ will be zero for the initial control points $p_{i}^{0}$ lying on the circle. If $d_{k}=0$ for large $\mathrm{k}$ then its mean scheme produces exact circle. The maximum deviation of an exact circle with the limiting circle can be calculated by $d_{k}$. Table 1 shows the deviation error of different limiting circles produced by proposed nonstationary subdivision schemes. The initial control points 
in Fig 1(a-d) are taken by the parametric equation of ellipse, parabola and hyperbola respectively and limit curve in Fig 1(a-d) are formed by applying proposed 4-point ternary subdivision scheme. Fig 2(a-f) shows the graphical representation of deviation error of proposed scheme at different level.

TABLE 1. DEVIATION ERROR OF PROPOSED SCHEMES. HERE N REPRESENTS INITIAL CONTROL POINTS

\begin{tabular}{|c|c|c|c|c|c|c|}
\hline Schemes & $N$ & Deviation Error & $N$ & Deviation Error & $N$ & Deviation Error \\
\hline 4-Point Proposed & 4 & 0.11427 & 5 & 0.05058 & 6 & 0.02540 \\
\hline 6-Point Proposed & 4 & 0.05075 & 5 & 0.02812 & 6 & 0.00562 \\
\hline
\end{tabular}

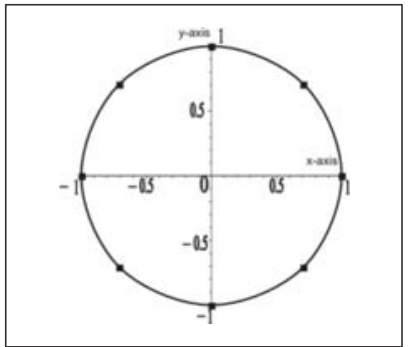

(a) CIRCLE

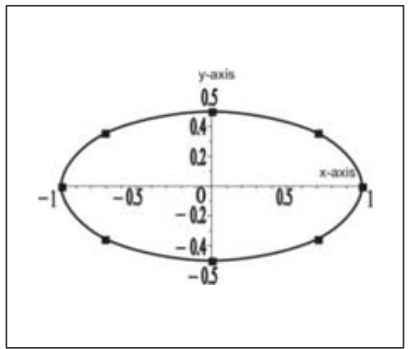

(b) ELLIPSE

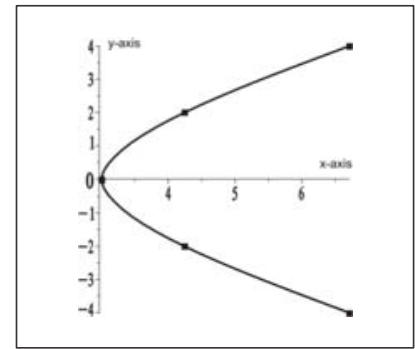

(c)HYPERBOLA



(d) PARABOLA

FIG. 1. SOLID BOXES INDICATE THE INITIAL CONTROL POINTS. SOLID CONTINUOUS CURVES ARE GENERATED BY PROPOSED 4-POINT TERNARY NON-STATIONARY INTERPOLATING SCHEME

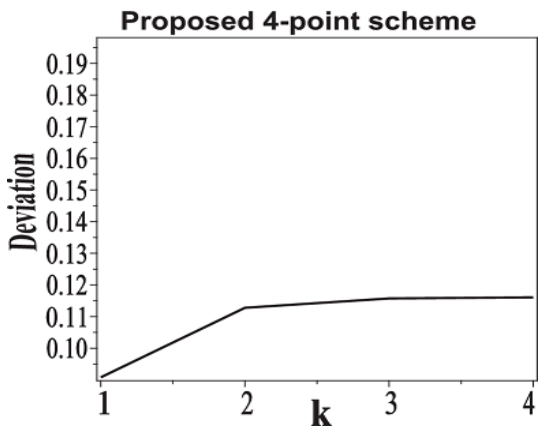

(a) 4 INITIAL POINTS

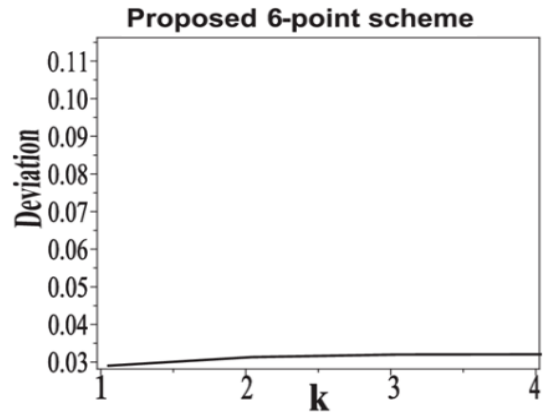

(d) 5 INITIAL POINTS

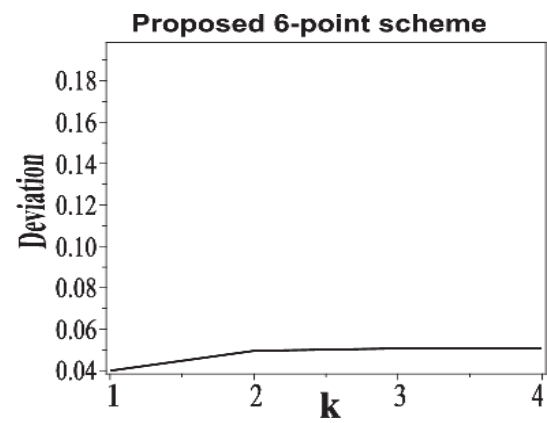

(b) 4 INITIAL POINTS

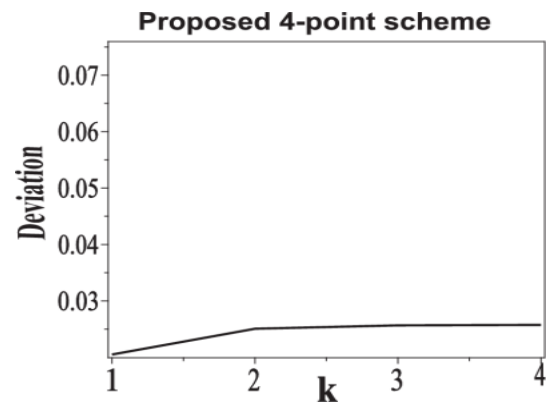

(e) 6 INITIAL POINTS

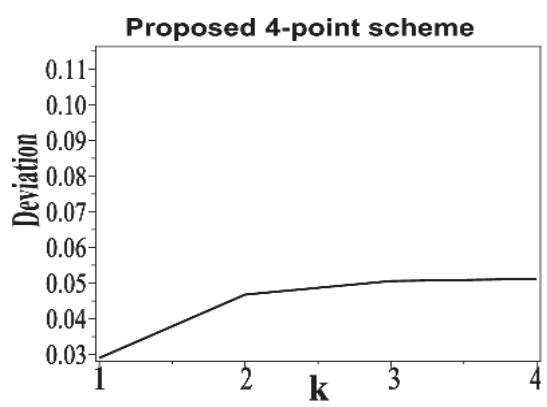

(c) 5 INITIAL POINTS

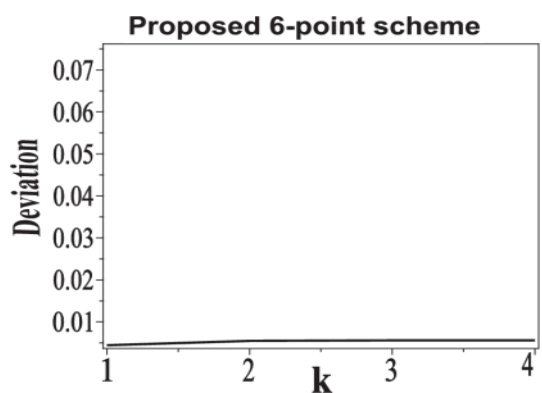

(f) 6 INITIAL POINTS

FIG. 2. GRAPHS SHOW THE DEVIATION AT FIRST, SECOND, THIRD AND FOURTH LEVEL USING 4, 5 AND 6 INITIAL DATA POINTS

Mehran University Research Journal of Engineering \& Technology, Volume 36, No. 4, October, 2017 [p-ISSN: 0254-7821, e-ISSN: 2413-7219] 


\section{CONCLUSION}

By using Lagrange identities we construct new families of univariate ternary non-stationary interpolating subdivision schemes for curve design with a single tension parameter which enable the scheme to produce more precise result. The proposed schemes are nonstationary counterpart of the existing stationary schemes, so the parametric ranges of continuity of proposed nonstationary schemes are same as that of the counter stationary schemes. In future work, proposed family of schemes can be extended for arbitrary topological surfaces.

\section{ACKNOWLEDGEMENT}

This work is supported by The Islamia University of Bahawalpur, Pakistan, and NRPU Project No. 3183 from Higher Education Commission (HEC) and also the Indigenous Ph. D 5000 Fellowship Program.

\section{REFERENCES}

Deslauriers, G., and Dubuc, S., "Symmetric Iterative Interpolation Processes”, Constructive Approximation, Volume 5, pp. 49-68, 1989.

Hassan, M.F., Ivrissimitzis, I.P., Dodgson, N.A., and Sabin, M.A., “An Interpolating 4-Point $C^{2}$ Ternary Stationary Subdivision Scheme”, Computer Aided Geometric Design, Volume 19, pp. 1-18, 2002.

Zheng, H., Hu, M., and Peng, G., "Constructing 2n-1-Point Ternary Interpolatory Subdivision Schemes by Using Variation of Constants”, Computational Intelligence and Software Engineering, [DOI: 10.1109/ CISE.2009.5364446], pp. 1-4, 2009 .

[4]

Lian, J.A.O., "On A-Ary Subdivision for Curve Design: 3-Point and 5-Point Interpolatory Schemes", Applications and Applied Mathematics, Volume 3, pp. 176-187, 2008.
[5] Siddiqi, S.S., and Rehan, K., "A Ternary Three Point Scheme for Curve Designing”, International Journal of Computer Mathematics, Volume 87, pp. 1709-1715, 2009.

[6] Siddiqi, S.S., and Ahmad, N., "A New Five Point Approximating Subdivision Scheme”, International Journal of Computer Mathematics, Volume 85, No. 1, pp. 65-72, 2008.

Bari, M., Bashir, R., and Mustafa, G., "3n-Point Quaternary Shape Preserving Subdivision Schemes”, Mehran University Journal of Engineering \& Technology, Volume 36, No. 3, pp. 489-500, Jamshoro, Pakistan, July, 2017.

Jena, M.K., Shunmugaraj,P., and Das, P.C., "A NonStationary Subdivision Schemefor CurveInterpolation”, ANZIAM Journal, Volume 44(E), pp. 216-235, 2003.

] Beccari, C., Casciola, G., and Romani, L., "A NonStationary Uniform Tension Controlled Interpolating 4-Point Scheme Reproducing Conics”, Computer Aided Geometric Design, Volume 24, No. 1, pp. 1-9, 2007.

Daniel, S., and Shunmugaraj, P., "Some Interpolating Non-Stationary Subdivision Schemes", International Symposium on Computer Science and Society, pp. 400-403,2011 [DOI 10.1109/ISCCS.2011.110].

[11] Bari, M., and Mustafa, G., "A Family of 4-Point n-Ary Interpolating Scheme Reproducing Conics”, American Journal of Computational Mathematics, Volume 3, pp. 217-221, 2013.

Mustafa, G., and Bari, M., "A New Class of Odd-Point Ternary Non-Stationary Schemes”, British Journal of Mathematics and Computer Science, Volume 4, pp. 133-152, 2014.

[13] Conti, C., Dyn, N., Manni, C., and Mazure, M.-L., "Convergence of Univariate Non-Stationary Subdivision Schemes via Asymptotic Similarity”, Computer Aided Geometric Design, Volume 37, pp. 1-8, 2015.

[14] Novara, P., and Romani, L., "Building Blocks for Designing Arbitrarily Smooth Subdivision Schemes with Conic Precision”, Journal of Computational and Applied Mathematics, Volume 279, pp. 67-79, 2015. 
[15] Mustafa, G., and Ashraf, P., "A Family of 4-Point OddAry Non-Stationary Subdivision Schemes”, SeMA Journal, Volume 67, pp. 77-91, 2015.

[16] Bari, M., "Stationary and Non-Stationary Subdivision Schemes and Their Applications", Ph.D. Thesis, Department of Mathematics, The Islamia University of Bahawalpur, Pakistan, 2016.
Dyn, N., and Levin, D., “Analysis of Asymptotically Equivalent Binary Subdivision Schemes”, Journal of Mathematical Analysis and Applications, Volume 193, pp. 594-621,1995.

[18] Aslam, M., Mustafa, G., and Ghaffar, A., "2n-1-Point Ternary Approximating and Interpolating Subdivision Schemes", Journal of Applied Mathematics, Volume 2011,Article ID 832630, pp. 13, 2011. 